\title{
Fibrous Phosphorus Quantum Dots for Cell Imaging
}

Pedro E. M. Amaral ${ }^{1 \ddagger}$, Donald C. Hall Jr. ${ }^{1,2 \ddagger}$, Rahul Pai, ${ }^{3}$ Jarosław Król ${ }^{2}$, Vibha Kalra, ${ }^{3}$

Garth D. Ehrlich², Hai-Feng Ji ${ }^{*}$

1Department of Chemistry, Drexel University, Philadelphia PA 19104

${ }^{2}$ Center for Advanced Microbial Processing, Drexel University, Philadelphia PA 19104

${ }^{3}$ Department of Chemical and Biological Engineering, Drexel University, Philadelphia

PA 19104

¥These authors contributed equally to this work

“Corresponding Author. Email Address: hj56@drexel.edu; Phone: 01-215-895-2562; Fax:

01-215-895-1265 


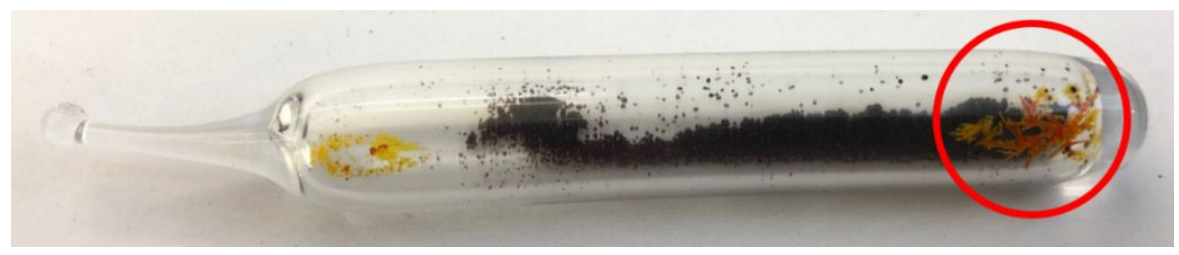

Figure S1. Ampoule after reaction at $580{ }^{\circ} \mathrm{C}$ containing FP crystals and a side product that can be easily dissolved and washed away with methanol (red circle).
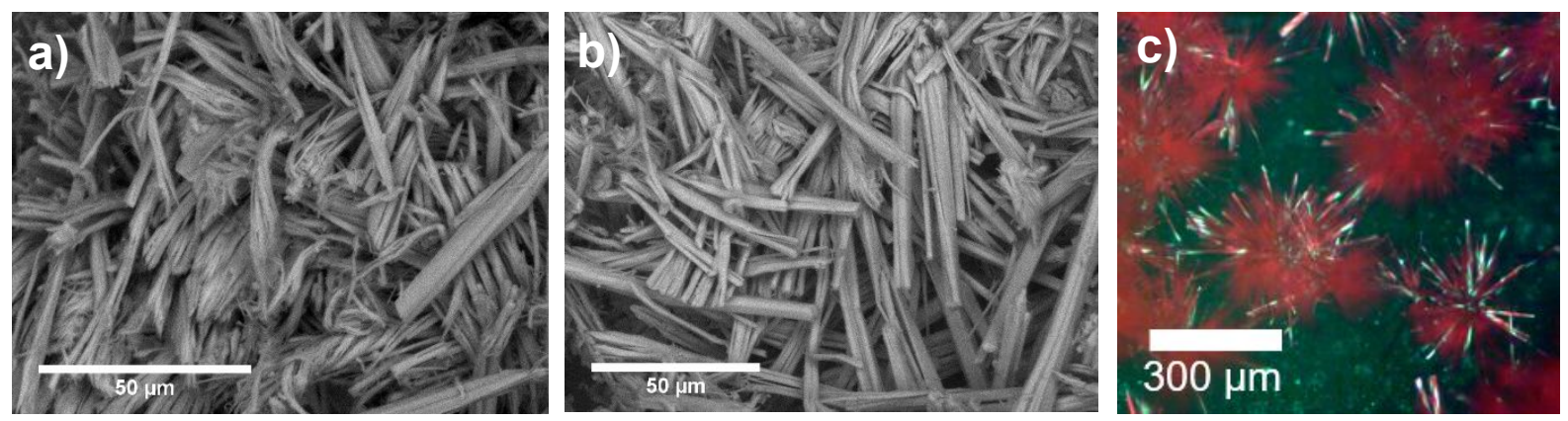

Figure S2. a) and b) SEM images of the synthesized FP crystals via the mineralizing agent approach. c) Optical image of the synthesized crystals. 


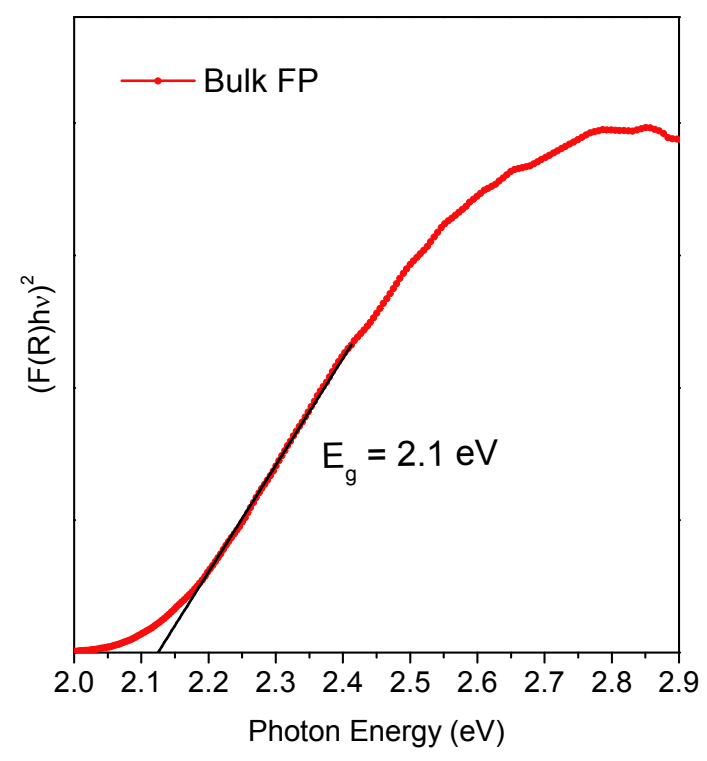

Figure S3. Tauc plot of bulk FP showing an optical band gap of $2.1 \mathrm{eV}$. The plot was obtaining by applying a transformation to the diffuse transmission spectrum of bulk BP. The diffuse transmission spectrum was obtained using an integrating sphere.
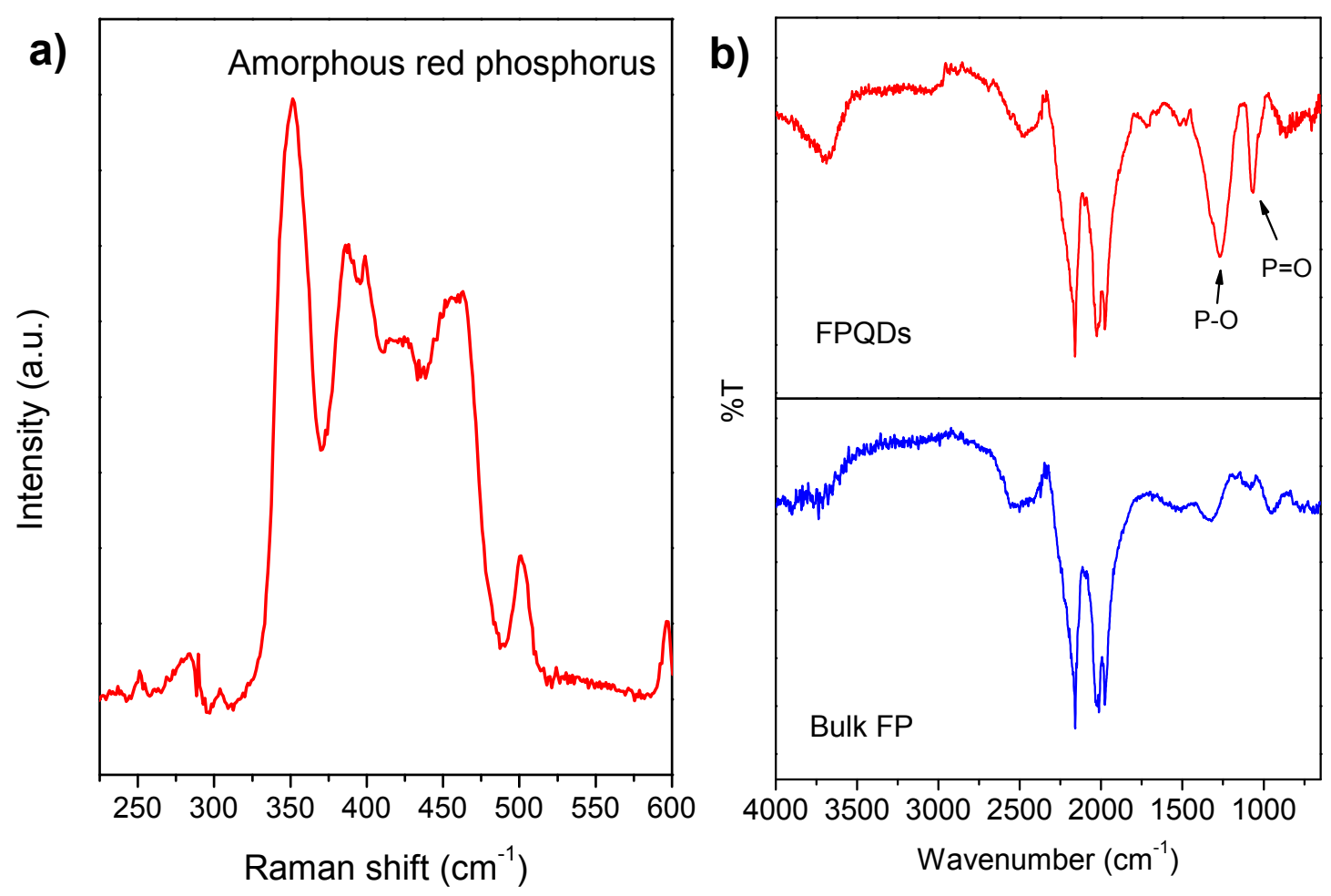

Figure S4. a) Raman spectrum of amorphous red phosphorus, b) FTIR spectra of bulk FP (bottom) and FPQDs (top). 

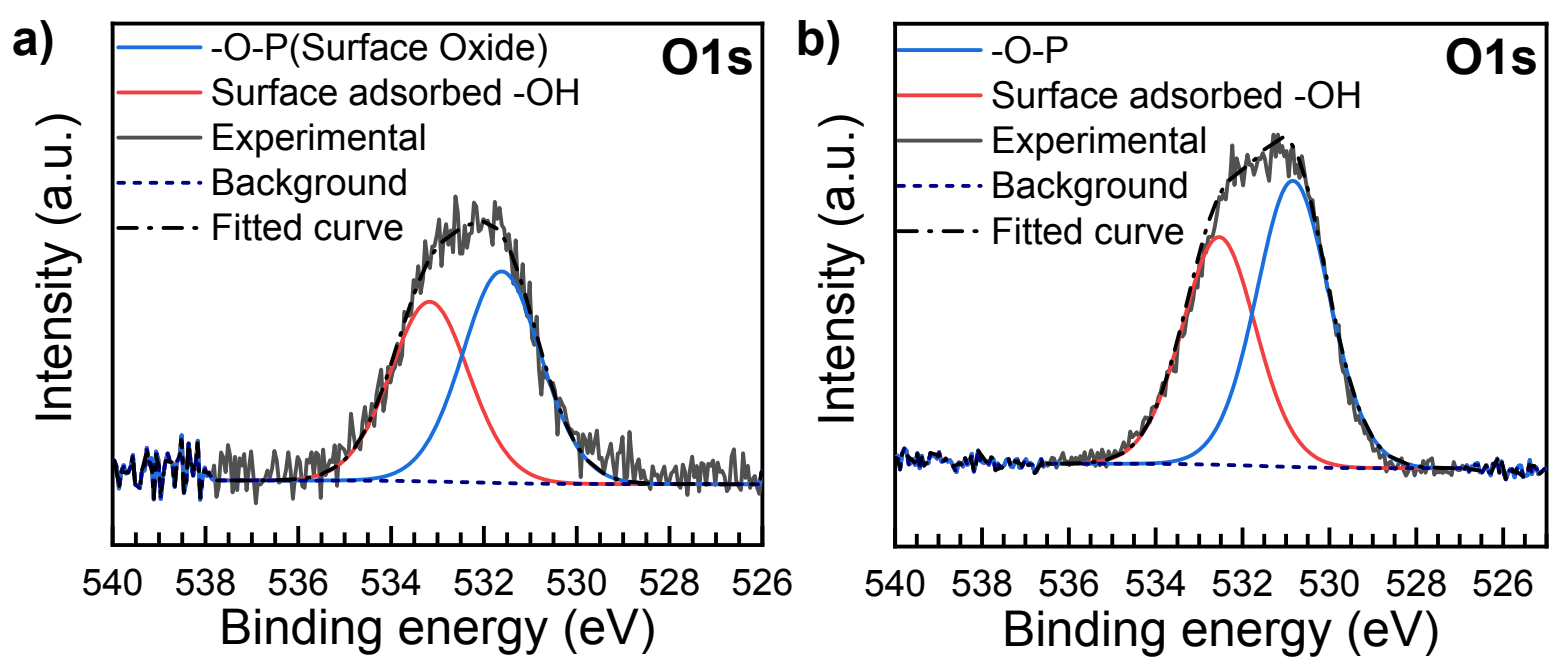

Figure S5. High-resolution XPS spectra for a) bulk FP, and b) FPQDs.

a)

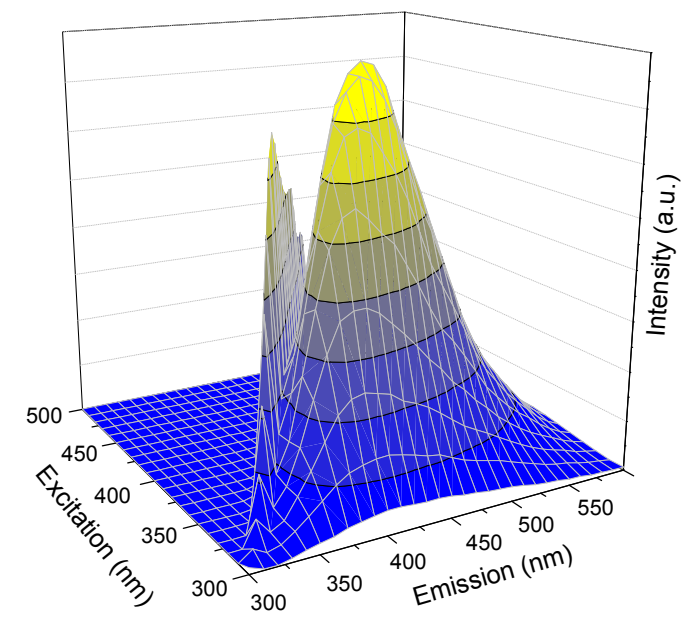

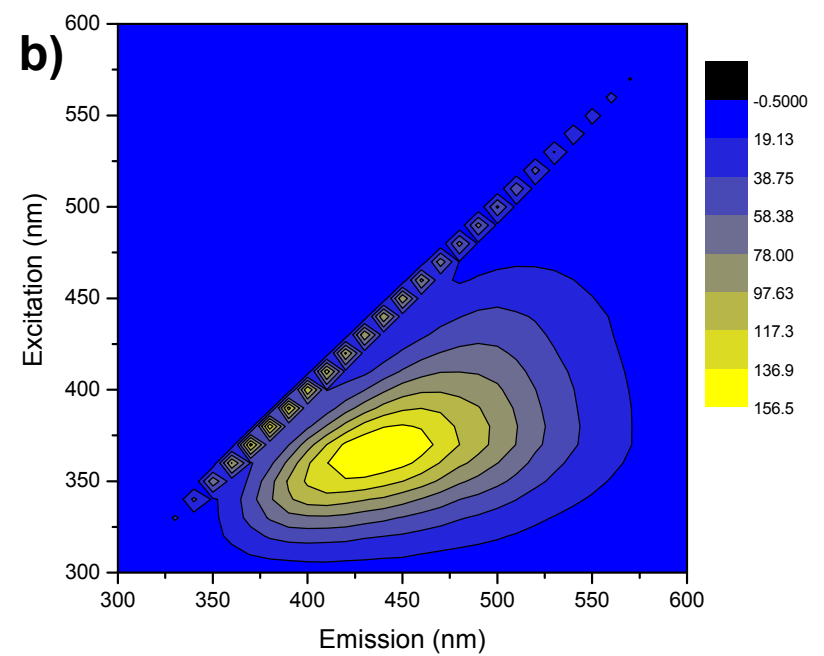

Figure S6. a) 3D fluorescence plot of the FPQDs, b) contour plot of the FPQDs fluorescence. 

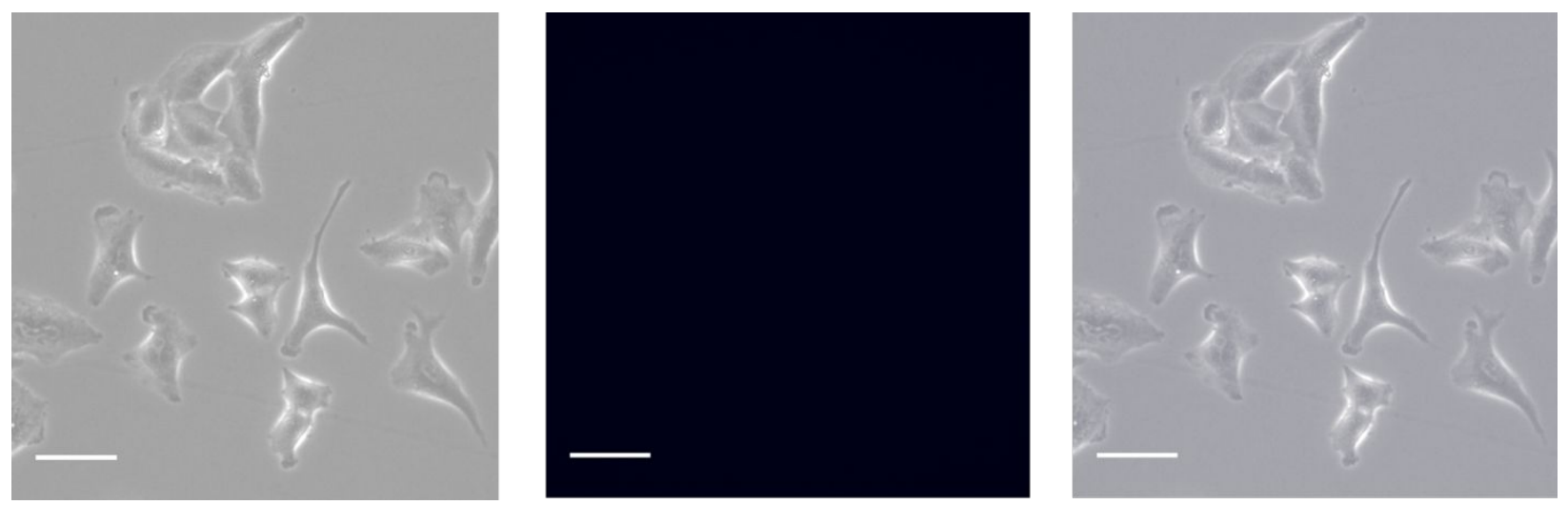

Figure S7. Bioimaging Control Human Adenocarcinoma Cells. a) brightfield images of untreated A549 human adenocarcinoma cells; b) fluorescent image of untreated cells (excitation wavelength $360 / 40 \mathrm{~nm}$, emission wavelength $460 / 50 \mathrm{~nm}$ ); c) merged brightfield and fluorescence images of cells (Scale bar $=50 \mu \mathrm{m})$. 